\title{
General Considerations of Noise in Microphone Preamplifiers
}

\author{
A. G. H. VAN DER DONK, J. A. VOORTHUYZEN and P. BERGVELD
}

University of Twente, P.O. Box 2I7, 7500 AE Enschede (The Netherlands)

\begin{abstract}
In this paper a study of the noise performance of electret microphone systems as a part of hearing aids is presented. The signalto-noise ratio of the microphone-preamplifier combination, containing a field-effect transistor (FET) and a high value resistive bias element in a hybrid configuration, is mainly determined by the noise generated in the preamplifier circuit.

A theoretical analysis of the noise sources in a source follower is given. The dominating noise sources are the channel noise of the FET, the thermal noise of the gate bias element, and finally the noise due to the gate leakage current of the FET and its package. It is shown that for the systems investigated, the noise performance does not depend on the choice of the amplifying device (JFET or MOSFET) itself, but only on its packages. Besides this, it is found that it is necessary to keep the parasitic capacitances as small as possible and to make the resistance of the bias element as large as possible.
\end{abstract}

\section{Introduction}

Recently, growing attention has been paid to the noise performance of electret microphone systems as part of hearing aids. The signal-to-noise ratio of the microphonepreamplifier combination is mainly determined by the noise generated in the preamplifier circuit. Until now only JFETs with a high input impedance have been used as the active part of the microphone preamplifier in a hybrid configuration, resulting in an $A$-weighted equivalent input noise of

$0924-4247 / 91 / \$ 3.50$ about $13 \mu \mathrm{V}$ typically and $19 \mu \mathrm{V}$ maximally [1]. Because it is possible that the preamplifier will be integrated with a silicon-based microphone [2] in the future, a CMOS preamplifier, which will have a very high input impedance, seems to be another possibility. Therefore the noise performance of microphone preamplifiers with JFETs has to be compared with that of preamplifiers with MOSFETs.

In this paper only the noise contribution of the FET and the bias element are considered. The noise caused by other components, like the impedance between source and ground or between drain and supply, will be neglected because of their minor influence.

As a starting point, in Section 2 a general model of the source follower with the capacitive microphone will be considered. In Section 3 some experimental results will be presented, and the noise-to-signal ratios of source followers with JFETs and MOSFETs will be compared and discussed.

\section{A General Model of the Microphone with the Source Follower}

In conventional hearing aids, a first-stage amplifier is used to transform the high capacitive output impedance of the electret microphone into a low impedance. Usually a source follower is applied. In an ideal situation, this has a unity voltage gain; in practice, however, an attenuation can be expected due to parasitic capacitances. In Fig. 1(a) a source follower with an enhancement-type p-channel MOSFET is drawn together with the capacitive microphone, which can be considered as a sound-pressure-sensitive voltage

(C) Elsevier Sequoia/Printed in The Netherlands 


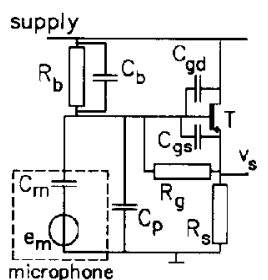

(a)

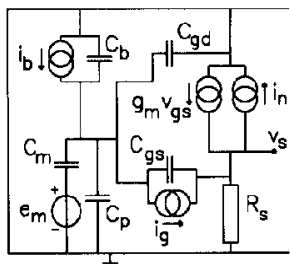

(b)

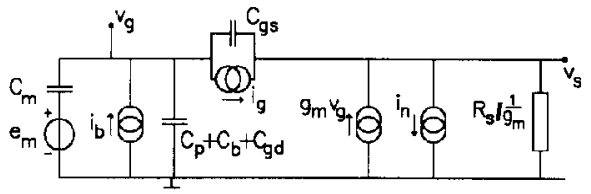

(c)

Fig. 1. (a) Capacitive microphone with a source follower with a FET $T$ and its capacitances, a resistive bias element $R_{\mathrm{b}}$ and leakage resistance $R_{\mathrm{g}}$ due to packaging. (b) Small-signal presentation of this circuit. (c) Simplified small-signal circuit.

source $e_{\mathrm{m}}$ with an output capacitance $C_{\mathrm{m}}$. A bias element having a resistance $R_{\mathrm{b}}$ and a (parasitic) capacitance $C_{b}$ is used to provide the gate with a well defined d.c. voltage. The FET is assumed to consist of an ideal transitor $\mathrm{T}$ without any capacitances, a gate-tosource capacitance $C_{\mathrm{gs}}$, a gate-to-drain capacitance $C_{\mathrm{gd}}$ and a gate-to-source leakage resistance $R_{\mathrm{g}}$. The source resistor $R_{\mathrm{s}}$ is chosen in such a way that its noise contribution can be neglected. Finally, a parasitic capacitance $C_{\mathrm{p}}$ due to wiring is shown in the Figure. If a JFET is used, the same drawing can be used, although in that case the bias element should be connected to ground.

Three noise generators will be considered: the noise current density $i_{\mathrm{b}}$ of the bias element parallel with the bias resistor, the FET noise current density $i_{\mathrm{n}}$ between drain and source, and the noise current density $i_{\mathrm{g}}$ due to the gate leakage resistance of the FET between gate and source. In Fig. 1(b) the smallsignal circuit is shown, also containing the three noise generators. To describe the smallsignal behaviour of the circuit, the resistors $R_{\mathrm{g}}$ and $\boldsymbol{R}_{\mathrm{b}}$ can be omitted, because their impedance is much larger than the impedance of the capacitances parallel with these resistors in the audio range of frequencies, as required to get a frequency-independent microphone signal transfer.

The FET is considered as a current source $g_{\mathrm{m}} v_{\mathrm{gs}}$, with $v_{\mathrm{gs}}$ the small-signal gate-to-source voltage and $g_{m}$ the channel transconductance $\left(\partial I_{\mathrm{d}} / \partial V_{\mathrm{gs}}\right)$ of the FET. The channel output conductance $\left(\partial I_{\mathrm{d}} / \partial V_{\mathrm{ds}}\right)$ of the FET can be neglected in the source follower configuration [3].

Analysing Fig. 1(c), which gives the simplified small-signal circuit, it is possible to calculate the contribution of the three noise sources and the signal source, and thus the noise-to-signal ratio, at the source. It can be found that the squared noise-to-signal ratio density can be expressed as [4]

$$
\begin{aligned}
& \frac{v_{\mathrm{s}}(n)^{2}}{v_{\mathrm{s}}\left(e_{\mathrm{m}}\right)^{2}}=\frac{\left(i_{\mathrm{b}}^{2}+i_{\mathrm{g}}^{2}\right)(1 / \omega)^{2}}{C_{\mathrm{m}}^{2} e_{\mathrm{m}}^{2}} \\
& +\frac{\left(C_{\mathrm{m}}+C_{\mathrm{p}}+C_{\mathrm{b}}+C_{\mathrm{gd}}+C_{\mathrm{gs}}\right)^{2}\left(i_{\mathrm{n}} / g_{\mathrm{m}}\right)^{2}}{C_{\mathrm{m}}^{2} e_{\mathrm{m}}^{2}}
\end{aligned}
$$

The equivalent input noise density $N_{\mathrm{i}}$ can be considered as a noise voltage source in series with the signal source $e_{\mathrm{m}} . N_{\mathrm{i}}^{2}$, which can be derived from eqn. (1) by multiplying the righthand part with $e_{\mathrm{m}}^{2}$, can be expressed as:

$$
\begin{array}{r}
N_{\mathrm{i}}^{2}=\frac{\left(C_{\mathrm{m}}+C_{\mathrm{p}}+C_{\mathrm{b}}+C_{\mathrm{gd}}+C_{\mathrm{gs}}\right)^{2}}{C_{\mathrm{m}}^{2}} \frac{i_{\mathrm{n}}^{2}}{g_{\mathrm{m}}^{2}} \\
+\frac{i_{\mathrm{b}}^{2}+i_{\mathrm{g}}^{2}}{\omega^{2} C_{\mathrm{m}}^{2}}
\end{array}
$$

The channel noise density of a FET can be expressed as [3]

$i_{\mathrm{n}}^{2}=g_{\mathrm{m}}^{2}\left(\frac{K}{f}+\alpha \frac{4 k T}{g_{\mathrm{m}}}\right)$

with $K$ a device- and bids-dependent parameter, $\alpha$ equal to 0.67 for JFETs and $\alpha \geqslant 0.67$ for MOSFETs, depending on the gate length [5].

The noise density due to the gate leakage current can be expressed as

$i_{\mathrm{g}}^{2}=\frac{4 k T}{R_{\mathrm{g}}}+2 q I_{\mathrm{g}}$

In this expression $R_{\mathrm{g}}$ is the gate leakage resistance, mainly caused by packaging of the bias element and the FET. The second part of eqn. (4) with $I_{g}$, the gate current, describes the shot noise, which occurs only in JFETs because $I_{\mathrm{g}}=0$ for MOSFETs. Finally the 
thermal noise density of the bias element is considered:

$i_{\mathrm{b}}^{2}=4 k T / R_{\mathrm{b}}$

Combining eqns. (2) to (5), the total equivalent input noise density of a JFET is

$$
\begin{aligned}
N_{\mathrm{ij}}^{2}= & \frac{\left(C_{\mathrm{m}}+C_{\mathrm{p}}+C_{\mathrm{b}}+C_{\mathrm{gd}}+C_{\mathrm{gs}}\right)^{2}}{C_{\mathrm{m}}^{2}} \\
& \times\left(\frac{K}{f}+0.67 \frac{4 k T}{g_{\mathrm{m}}}\right)+\left(\frac{1}{R_{\mathrm{b}}}+\frac{1}{R_{\mathrm{g}}}\right) \frac{4 k T}{\omega^{2} C_{\mathrm{m}}^{2}} \\
& +\frac{2 q I_{\mathrm{g}}}{\omega^{2} C_{\mathrm{m}}^{2}}
\end{aligned}
$$

and of a MOSFET

$$
\begin{aligned}
& N_{\mathrm{iM}}^{2}=\frac{\left(C_{\mathrm{m}}+C_{\mathrm{p}}+C_{\mathrm{b}}+C_{\mathrm{gd}}+C_{\mathrm{gs}}\right)^{2}}{C_{\mathrm{m}}^{2}} \\
& \times\left(\frac{K}{f}+\alpha \frac{4 k T}{g_{\mathrm{m}}}\right)+\left(\frac{1}{R_{\mathrm{b}}}+\frac{1}{R_{\mathrm{g}}}\right) \frac{4 k T}{\omega^{2} C_{\mathrm{m}}^{2}}
\end{aligned}
$$

It is desirable to analyse eqns. (6) and (7) for audio frequencies. Because they are frequency dependent, a simple comparison is not always possible. In order to analyse the total noise performance of the circuit in a hearing aid application, eqns. (6) and (7) have to be filtered by the so-called $A$-weighting function [6], which represents the frequency-dependent sensitivity of the human ear:

$$
N_{\mathrm{i}}(A)^{2}=\int_{f_{1}}^{f_{2}} N_{\mathrm{i}}^{2} A_{\mathrm{w}}^{2} \mathrm{~d} f
$$

with $f_{1}=1 \mathrm{~Hz}, f_{2}=100 \mathrm{kHz}$

By using eqn. (8) for source followers with JFETs and MOSFETs, in combination with eqns. (6) and (7) respectively, both configurations can be compared.

\section{Experimental}

In order to compare eqns. (6) and (7), a few device parameters have to be measured. For the measurements, $\mathbf{n}$-channel JFETs were used, which were fabricated especially for electret microphone preamplifiers. The $\mathrm{p}$ channel MOSFETs used for these experiments were fabricated at the CMOS production facility at the University of
Twente. For the noise measurements a capacitor of $2 \mathrm{pF}$ was used instead of a capacitive microphone.

\subsection{Channel Noise $i_{n}$ of JFETs and MOSFETS}

In order to determine the FET noise $i_{n}^{2}$, the noise of source followers is measured with a set-up as shown in Fig. $2\left(R_{\mathrm{b}}=0\right)$. In this circuit the output noise density can be expressed as [4]

$N_{\mathrm{o}}^{2}=\left(\frac{R_{\mathrm{s}}}{1+g_{\mathrm{m}} R_{\mathrm{s}}}\right)^{2} i_{\mathrm{n}}^{2}=\frac{H_{\mathrm{A}}^{2} i_{\mathrm{n}}^{2}}{g_{\mathrm{m}}^{2}}$

Figure 3 shows the measured output noise density spectrum of a JFET (curve a) and a MOSFET (curve b) source follower. As can be seen in these plots, there is no significant difference in flicker noise as well as in thermal noise between JFETs and p-channel MOSFETs.

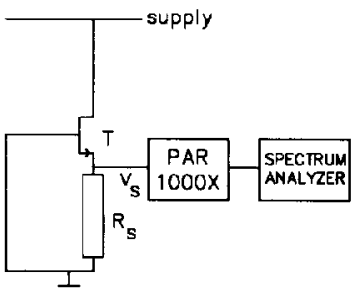

Fig. 2. Measurement set-up for the channel noise of a FET,

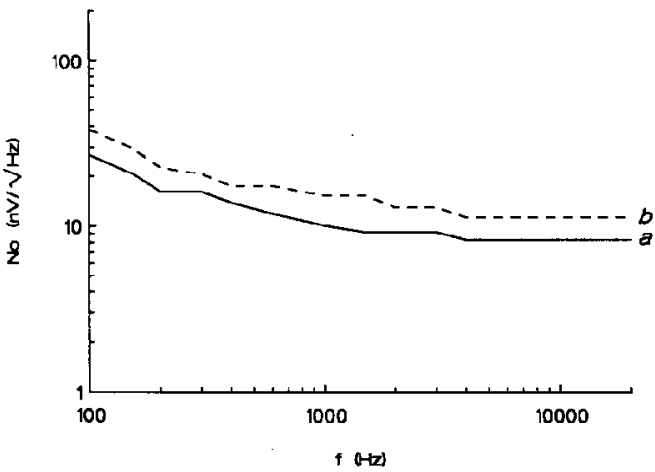

Fig. 3. Measured channel noise density as a function of frequency of (a) a JFET and (b) a MOSFET with a gate oxide thickness $t_{\mathrm{ox}}$ of $25 \mathrm{~nm}$, a gate $L$ of $5 \mu \mathrm{m}$ and a gate $W$ of $440 \mu \mathrm{m}$. Drain current $I_{\mathrm{d}}=30 \mu \mathrm{A}$. 


\subsection{Noise $i_{h}$ of the Bias Element}

In a hybrid configuration with a JFET, a thick-film resistor of several G $\Omega$ is commonly used as the bias element. If the resistor exhibits only thermal noise, its noise can be expressed according to eqn. (5). However, if the preamplifier is realized in a CMOS process, the bias element can be realized in the same process. In that case it is not desirable to use a resistor as a bias element, because it will consume too much space on the chip. A zero-biased diode or a switched-off MOSFET will be a good alternative. The shot-noise current density of a zero-biased diode can be described by

$i_{\mathrm{D}}^{2}=\frac{4 k T}{r_{\mathrm{D}}}$

and can be considered to be the thermal noise of a resistor with a small-signal resistance $r_{\mathrm{D}}$. I ssing a parameter analyser, an average value of about $100 \mathrm{G} \Omega$ for $r_{\mathrm{D}}$ has been measured for diodes fabricated at the University of Iwente. The thick-film resistor as used by Microtel [1] in their hearing aid microphones had a value of about $6 \mathrm{G} \Omega$.

Using these results, it can be concluded that the noise contribution of the bias element will be much larger when a thick-film resistor is used than when a diode is used. Unfortunately, it is not possible to measure the noise of the bias elements separately.

\subsection{Noise $i_{\mathrm{g}}$ Caused by the Gate Leakage Current}

Measuring the d.c. gate leakage current $I_{\mathrm{g}}$ of the JFET with a parameter analyser for several devices, an average value of $0.5 \mathrm{pA}$ was observed.

Using a General Radio 1621 impedance measurement system, the frequency dependence of the resistive and the capacitive part of the JFET gate admittance and the admittance of the bias resistor were measured. The same measurement was done for MOSFETs with a bias diode. The capacitances were found to be $5.5 \mathrm{pF}$ for JFETs and $7 \mathrm{pF}$ for MOSFETs and were frequency independent.

The average values of the resistive part of

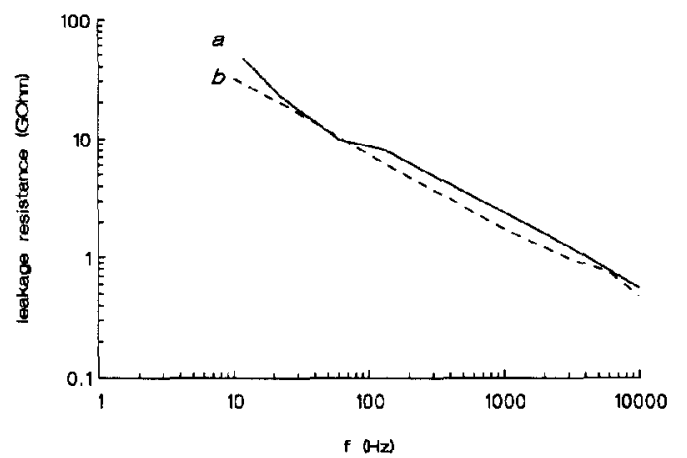

Fig. 4. Measured leakage resistance as a function of frequency of (a) a JFET with thick-film bias resistor, (b) a MOSFET ( $t_{\mathrm{ox}}=25 \mathrm{~nm}, L=5 \mu \mathrm{m}, W=440 \mu \mathrm{m}$ ) with bias diode.

the gate impedance for JFETs as well as MOSFETs have also been measured. For the JFET, this resistance is considered to consist of an ideal, frequency-independent resistor $R_{\mathrm{b}}$ of $6 \mathrm{G} \Omega$ parallel with a frequency-dependent resistance $R_{\mathrm{g}}$, of which the experimental results are plotted in Fig. 4 (curve a). Note that for frequencies higher than approximately $100 \mathrm{~Hz}$, the leakage resistance $R_{\mathrm{g}}$ is smaller than the $6 \mathrm{G} \Omega$ bias resistor $R_{\mathrm{b}}$. Curve b shows the measured frequency-dependent leakage resistance of the circuit with a MOSFET. If these resistances exhibit thermal noisc, their contribution to the equivalent input noise voltage density can also be calculated for both source followers, as shown in Fig. 5, curves a and b respectively. In Fig. 5 (curve c) the contribution of the shot noise to the equivalent input noise density of the source follower with a JFET is shown, as calculated with eqn (6). According to this Figure, the contribution of the shot noise of the JFET can be neglected in comparison with the contribution of the thermal noise due to the gate leakage resistance of the source follower.

\subsection{The Equivalent Input Noise Density of Source Followers with JFETs and MOSFETS}

Considering the results presented in Figs. 3 and 5 and eqns. (6) and (7), it is possible to 


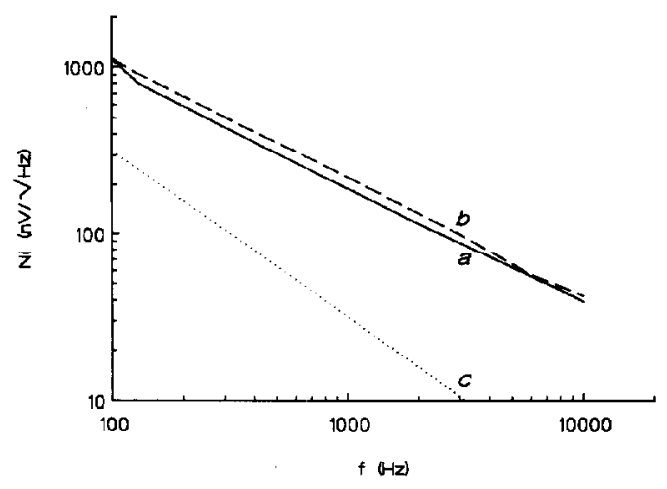

Fig. 5. Calculated thermal noise density as a function of frequency of (a) a JFET with thick-film resistor and (b) a MOSFET ( $t_{\mathrm{ox}}=25 \mathrm{~nm}, L=5 \mu \mathrm{m}, W=440 \mu \mathrm{m}$ ) with bias diode, and calculated shot noise of the JFET $\left(I_{\mathrm{g}}=0.5 \mathrm{p} \Gamma\right)$.

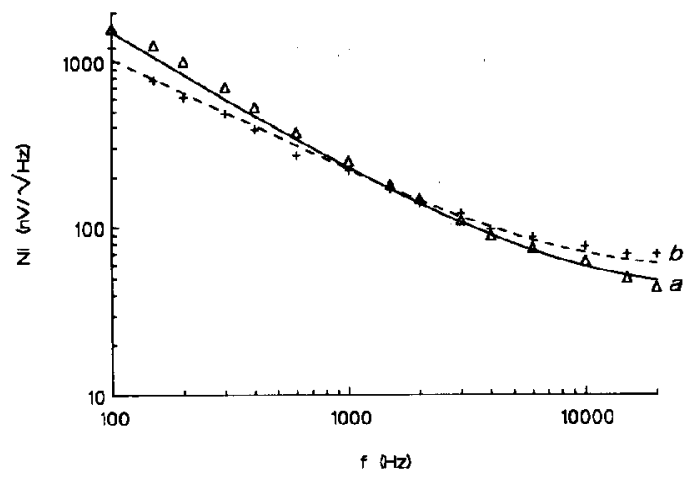

Fig. 6. Equivalent input noise density as a function of frequency of a capacitive signal source with a source follower $\left(I_{\mathrm{d}}=30 \mu \mathrm{A}\right)$. (a) Calculated, with a JFET and a thick-film bias resistor of $6 \mathrm{G} \Omega$, based on the experimental results of Figs. 3 (curve a) and 4 (curve a). (b) Calculated, with a MOSFET and a bias diode, based on the experimental results of Figs. 3 (curve b) and 4 (curve b). ( +) Measured with a JFET and a thick-film resistor of $6 \mathrm{G} \Omega$. ( $\triangle)$ Measured with a MOSFET ( $t_{\mathrm{ox}}=25 \mathrm{~nm}, L=5 \mu \mathrm{m}, W=440 \mu \mathrm{m}$ ) and a bias diodc.

calculate the equivalent input noise spectrum of source followers with JFETs as well as with MOSFETs, as shown in Fig. 6 curves a and $b$ respectively. With a spectrum analyser the equivalent input noise spectra of source followers with JFETs and MOSFETs have also been measured, as denoted in Fig. 6 by $\Delta$ and + respectively. Comparing the measured and the calculated plots, it can be concluded

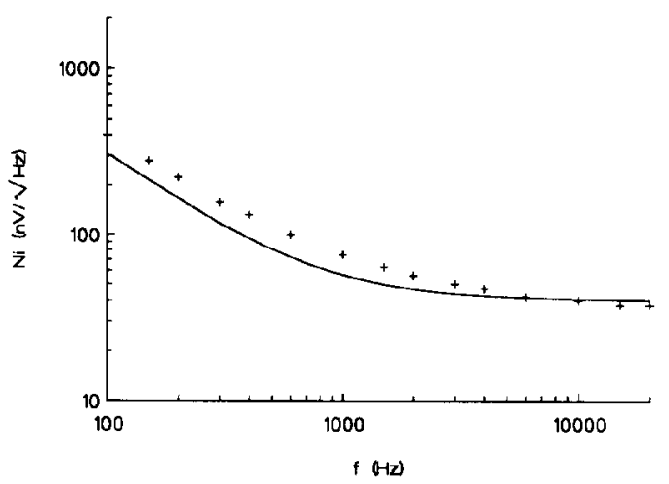

Fig. 7. $(+)$ Measured equivalent input noise density of a completely integrated capacitive signal source with a source follower with a MOSFET $\left(t_{\mathrm{ox}}=25 \mathrm{~nm}\right.$, $L=10 \mu \mathrm{m}, W=1000 \mu \mathrm{m})$ and a bias diode. Solid curve: calculated equivalent input noise density of a capacitive signal source with a source follower with a MOSFET ( $\left.t_{\mathrm{ox}}=25 \mathrm{~nm}, L=10 \mu \mathrm{m}, W=1000 \mu \mathrm{m}\right)$ and a bias resistor with $R_{\mathrm{b}}=100 \mathrm{G} \Omega, C_{\mathrm{b}}+C_{\mathrm{p}}+C_{\mathrm{gs}}+$ $C_{\mathrm{gd}}=1.75 \mathrm{pF}, I_{\mathrm{d}}=30 \mu \mathrm{A}$. The flicker noise of the MOSFET is based on the results in Fig. 3.

that the previous considerations concerning the three noise sources in source followers seem to be in good agreement with the experimental results. On the other hand, there is no significant difference in noise behaviour between source followers with JFETs and source followers with MOSFETs.

Finally, by comparing Figs. 5 and 6 it can be concluded that the dominant noise source is the frequency-dependent leakage resistance $R_{\mathrm{g}}$ for both types of source followers. In order to find the origin of this leakage resistance, special chips were made containing the circuit of Fig. 1 completely integrated. Because in this case the gate is not connected to a terminal of the package, the leakage resistance $R_{\mathrm{g}}$ should be infinite if it is a package resistance and still have a finite value if it is the resistance of the FET or the bias element itself. This measurement was only done with CMOS circuits, fabricated at the University of Twente. In Fig. 7 the measured equivalent input noise of this circuit is shown as a function of frequency (curve marked with + ), together with the equivalent input noise calculated with eqn. (7) with $R_{\mathrm{b}}=\infty$ and $R_{\mathrm{b}}=100 \mathrm{G} \Omega$ (solid curve). Note that the 
difference between the experimental and calculated results in Fig. 7 can be explained by the actual value of $R_{\mathrm{b}}$, which could be smaller than $100 \mathrm{G} \Omega$ in the completely integrated devices. For the $A$-weighted equivalent input noise voltage, a value of about $5 \mu \mathrm{V}$ r.m.s. was measured. Comparing Fig. 7 with Fig. 6 (curve b), it appears that the equivalent input noise is reduced significantly due to the absence of the gate leakage resistance, which is apparently caused by the package of the MOSFET and the bias diode.

\section{Discussion and Conclusions}

It can be concluded that there is no significant difference in noise performance between a source follower with a JFET and one with a p-channel MOSFET, as long as the circuit is applied in a hybrid configuration. The thermal noise due to the frequency-dependent leakage resistance of the package is dominant in both types of circuits. However, if the circuit can be completely integrated with the microphone, this important noise source will be eliminated, resulting in an $A$-weighted equivalent input noise of about $5 \mu \mathrm{V}$ for MOSFETs, being a significant improvement as compared with the specifications of Microtel of $13 \mu \mathrm{V}$ [1]. In this case the noise produced by the bias element will dominate and therefore its resistance should be chosen to be as large as possible to reduce its noise contribution. However, there will be an upper restriction for its value when a JFET is used, because the leakage current $I_{\mathrm{g}}$ of the JFET will cause a voltage drop across the bias element, resulting in a bias of the JFET which depends strongly on $I_{\mathrm{g}}$. In the case when a MOSFET is used, which has a zero leakage current, a much larger bias resistor can be used, while the voltage drop across the bias element still can be neglected. Therefore the use of MOSFETs in a microphone preamplifier may offer a significant advantage, compared to the use of JFETs.

\section{References}

I Microtel, specification of electret microphone MODEL 32, Amsterdam, June 1986.

2 J. A. Voorthuyzen, P. Bergveld and A. J. Sprenkels, Semiconductor-based electret sensors for sound and pressure, IEEE Trans. Electr. Insul., EI-24 (1989) 267-276.

3 P. R. Gray and R. G. Meyer, Analysis and Design of Analog Integrated Circuits, Wiley, New York, 2nd edn., 1986, pp. 56, 69, 729, 646.

4 A. G. H. van der Donk, J. A. Voorthuyzen and P. Bergveld, Optimal design of electret microphone MOSFET preamplifier, J. Acoust. Soc. Am., submitted for publication.

5 R. P. Jindall, General noise considerations of gigabitrate NMOSFET front-end design for optical-fiber communication systems, IEEE Trans. Electron Devices, ED-34 (1987) 305-309.

6 W. R. Bevan, R. B. Schulein and C. E. Seeler, Design of a studio-quality condenser microphone using electret technology, J. Audio Eng. Soc., 26 (1978) 947 957. 